\title{
MECHANISM OF SORPTION EQUILIBRIUM IN THE RECOVERY OF ZINC, CALCIUM, AND MAGNESIUM FROM WASTE WATER BY THE USE OF IMINODIACETATE RESINS
}

\author{
K. L. Timofeev and S. S. Naboichenko
}

UDC 669.5:628.349.081.312.3

A study is made of the laws that govern the sorptive equilibrium of zinc, calcium, and magnesium in aqueous solutions on iminodiacetate commercial resin Lewatit TP 207. The relations that are obtained show that the cation sorption process is described by the Langmuir and Freundlich models. The selectivity series $\mathrm{Zn}^{2+}>\mathrm{Ca}^{2+}>\mathrm{Mg}^{2+}$ was established based on determination of the affinity constants in the corresponding equations. Thus, the resin can be used to remove zinc cations from waste water even when it contains high concentrations of hardness ions.

Keywords: iminodiacetate resin, sorption, equilibrium, selectivity, waste waters.

Mine water that contains ore-forming elements - including elements which are toxic - poses a serious problem for mining companies in the nonferrous metallurgy industry. Projections show that the amount of zinc in mine water and quarry water will not decrease over the coming decade, which points to the importance of developing methods of removing zinc from industrial effluents [1].

Laboratory tests have determined that the iminodiacetate ion-exchange resin Lewatit TP 207 Monoplus is an effective sorbent of copper and zinc despite the predominant concentration of hardness ions in the solution [2]. The concentration of nonferrous metals in waste water after it is passed through this material satisfies health standards. It was determined that the impurity which limits the sorption process is zinc, since copper is effectively extracted by ion-exchange resins that contain amino groups [3]. There have only been a few studies of ion exchange involving cations of zinc and hardness ions. The investigation being discussed in this article examined the sorption equilibrium mechanism in the absorption of zinc, magnesium, and calcium from model aqueous solutions.

Describing exchange equilibrium in complex-forming ion-exchange resins is a component part of the larger problem of studying the equilibrium of such resins. In the general case, describing exchange equilibrium reduces to derivation of the equation of the ion-exchange isotherm that characterizes the state of ion-exchange equilibrium at a constant temperature. This isotherm links the numbers of ions in each phase and makes it possible to determine the selectivity of a given ionexchange resin [4].

Before tests were performed, iron was washed from the ampholyte with the use of three unit volumes of 5\% hydrochloric acid and six unit volumes of 5\% caustic soda. The ion-exchange resin that was prepared in $\mathrm{Na}^{+}$was washed to $\mathrm{pH}=5.5$ and stored under a layer of distilled water.

The initial solutions were prepared from chemically pure reagents $\mathrm{ZnSO}_{4} \cdot \mathrm{H}_{2} \mathrm{O}, \mathrm{MgSO}_{4} \cdot 7 \mathrm{H}_{2} \mathrm{O}$, and $\mathrm{CaCl}_{2}$ by dissolving them in distilled water. The initial concentration of the metals in each solution was within the range $0.5-23.5 \mathrm{mg}$-eqv/ $/ \mathrm{dm}^{3}$; the $\mathrm{pH}$ of the test solutions was 6.0 .

Yeltsin Ural Federal University (UrFU), Yekaterinburg, Russia: e-mail: k.timofeev@elem.ru. Translated from Metallurg, No. 2, pp. 35-38, February, 2012. Original article submitted October 29, 2012. 


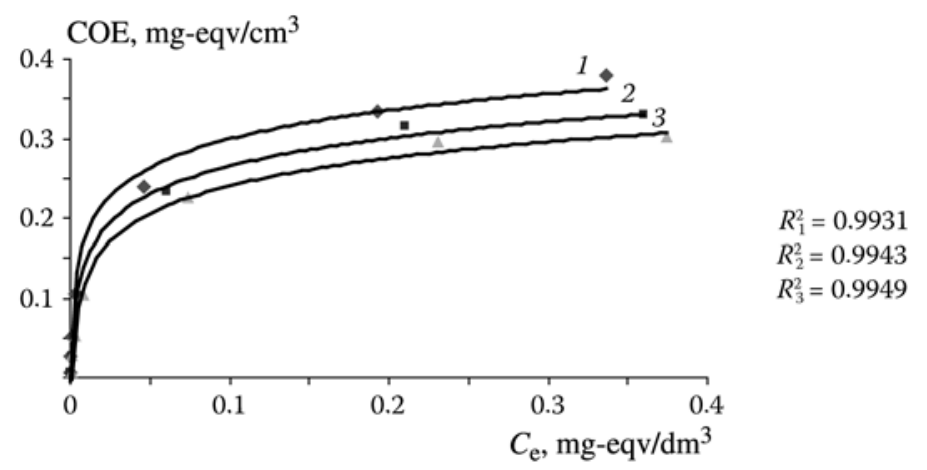

Fig. 1. Sorption isotherms of the elements $\left(C=0.5 \mathrm{mg}\right.$-eqv/ $\left.\left.\mathrm{dm}^{3}\right): 1\right)$ zinc; 2) calcium; 3) magnesium.

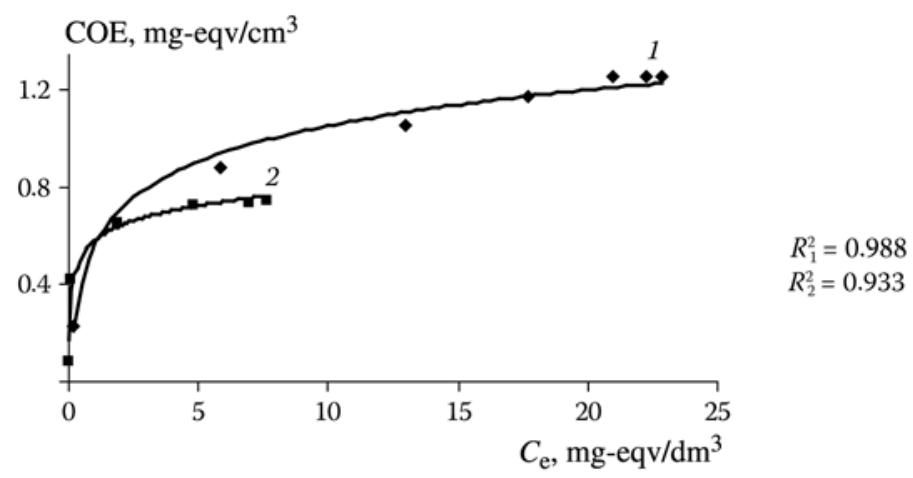

Fig. 2. Sorption isotherms: 1$)$ calcium $\left.\left(C=23.5 \mathrm{mg-eqv} / \mathrm{dm}^{3}\right) ; 2\right)$ magnesium $\left(C=8.5 \mathrm{mg}\right.$-eqv $\left./ \mathrm{dm}^{3}\right)$.

The variable-volume method was used in studying the output sorption curves obtained for a temperature of $25^{\circ} \mathrm{C}$. Weighed portions of the ion-exchange resin with a volume of $1 \mathrm{~cm}^{3}$ were dewatered in air to the dry-air state and added to the test solution. The S:L ratio (the ion-exchange-resin/solution ratio) was within the range 1:10-1:2000. The total time that the resin was in contact with the solution was $60 \mathrm{~h}$. Two hours of this time was spent mixing the solution in a PU EKROS 6300M shaker at a frequency of $30 \mathrm{~min}^{-1}$. The concentrations of the elements in the equilibrium solutions were determined by atomic-absorption spectrometry (Perkin Elmer AAnalyst 400). The results were analyzed with the use of Microsoft Excel 2007.

The character of the output sorption curves for zinc, calcium, and magnesium formally correspond to the Langmuir adsorption isotherms when the concentrations of these metals in the initial solution is $0.5 \mathrm{mg}$-eqv/dm ${ }^{3}$ (Fig. 1). The capacity of the ion-exchange resin increases with an increase in the equilibrium concentration of the elements in the solution.

The character of the output sorption curve remains with same with an increase in the content of calcium ions in the solution from 0.5 to $23.5 \mathrm{mg}$-eqv $/ \mathrm{dm}^{3}$ but the capacity of the ion-exchange resin increases (line 1 in Fig. 1). A similar pattern is seen in regard to magnesium: an increase in its concentration in the initial solution from 0.5 to $8.5 \mathrm{mg}$-eqv/ $/ \mathrm{dm}^{3} \mathrm{does}$ not alter the form of the sorption isotherm but does increase the capacity of the resin (line 2 in Fig. 2).

The character of all the output sorption curves that were obtained illustrates the selectivity of the resin in the recovery of the investigated elements from monosolutions. The high degree of reliability of the approximation $\left(R^{2}>0.9\right)$ based on the logarithmic (ln) trend line confirms that the output curve is of the Langmuir type. 


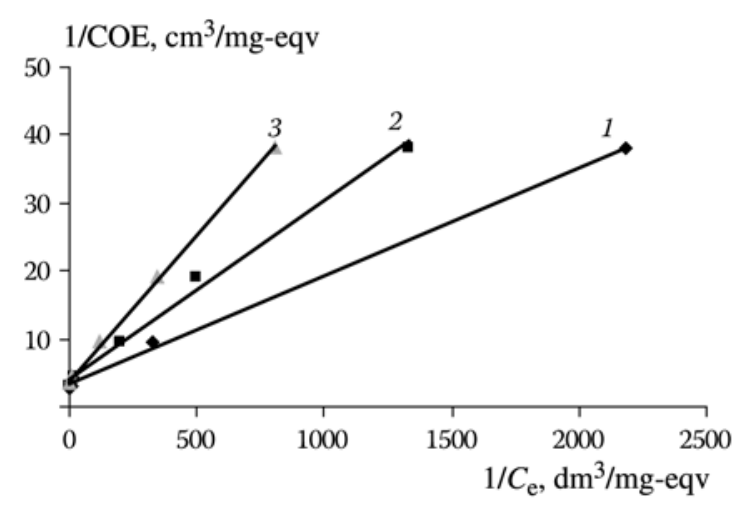

$a$

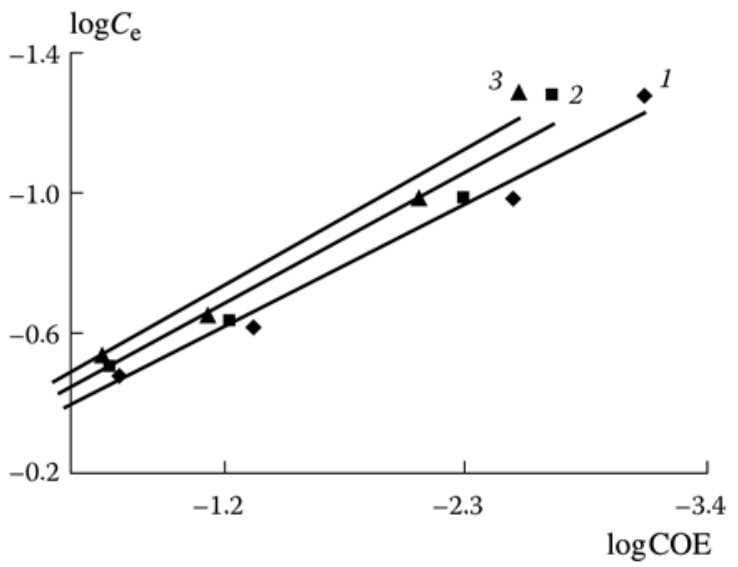

b

Fig. 3. Sorption isotherms of the elements: $a)$ in accordance with the Langmuir equation $\left(C=0.5 \mathrm{mg}\right.$-eqv/dm $\left.{ }^{3}\right)$; $b)$ in accordance with the Freundlich equation $\left(C=0.5 \mathrm{mg}_{\text {-eqv}} / \mathrm{dm}^{3}\right)$ : 1) zinc; 2) calcium; 3) magnesium.

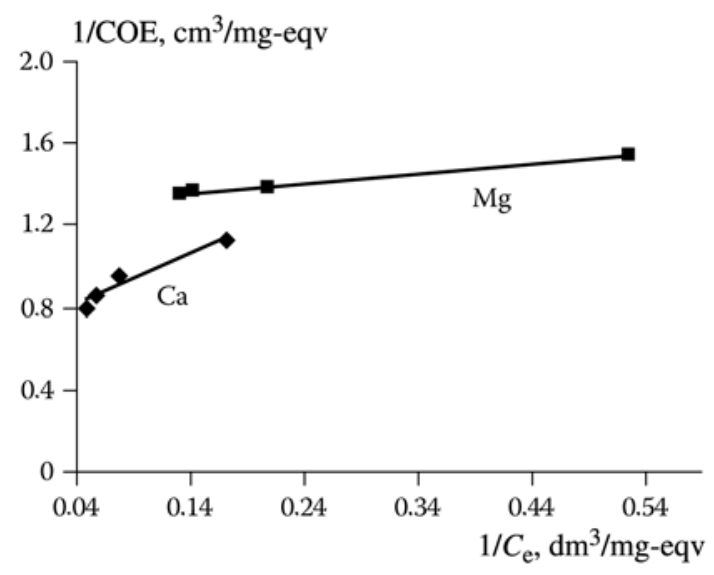

a

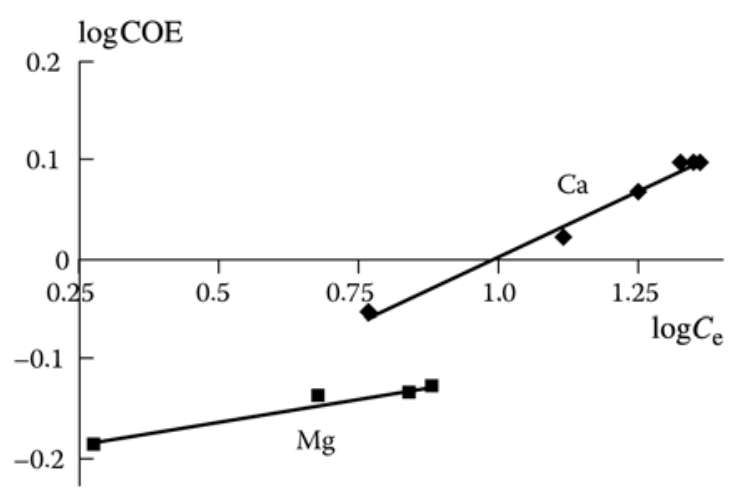

b

Fig. 4. Sorption isotherms of calcium at $C=23.5 \mathrm{mg}$-eqv $/ \mathrm{dm}^{3}$ and of magnesium at $C=8.5 \mathrm{mg}$-eqv/dm ${ }^{3} \mathrm{based}$ on the Langmuir equation $(a)$ and the Freundlich equation $(b)$.

We used the Langmuir and Freundlich adsorption models to describe the sorption process quantitatively. These models can also be successfully used to obtain the equilibrium characteristics of ion-exchange resins [3]. The Langmuir model of monomolecular sorption was developed for the following conditions [5]:

1) sorption is localized on individual sorption centers, each of which interacts only with one molecule of the adsorbate; this results in the formation of a monomolecular layer;

2) adsorption centers are energetically equivalent; and

3 ) the adsorbed molecules do not interact with one another.

The Langmuir model is described by the following equation [6]:

$$
C_{\mathrm{e}} / Q_{\mathrm{e}}=C_{\mathrm{e}} / Q_{s}+1 / K_{\mathrm{ads}} Q_{s}
$$

where $C_{\mathrm{e}}$ is the equilibrium concentration of ions in the solution, mg-eqv/dm ${ }^{3} ; Q_{\mathrm{e}}(\mathrm{COE})$ is the capacity of the ion-exchange 
TABLE 1. Sorption Parameters of the Elements $\left(C=0.5 \mathrm{mg}\right.$-eqv $\left./ \mathrm{dm}^{3}\right)$

\begin{tabular}{|c|c|c|c|c|c|c|}
\hline \multirow{2}{*}{ Element } & \multicolumn{3}{|c|}{ Langmuir equation } & \multicolumn{3}{c|}{ Freundlich equation } \\
\cline { 2 - 7 } & $Q_{s}$, mg-eqv/cm & $K_{\text {ads }}$ & $R^{2}$ & $K_{s}$ & $n$ & $R^{2}$ \\
\hline $\mathrm{Zn}$ & 0.30 & 211.25 & 0.99 & -0.24 & 0.32 & 0.98 \\
\hline $\mathrm{Ca}$ & 0.26 & 146.15 & 0.99 & -0.27 & 0.34 & 0.96 \\
\hline $\mathrm{Mg}$ & 0.27 & 86.74 & 0.99 & -0.3 & 0.35 & 0.96 \\
\hline $\mathrm{Ca}\left(23.5 \mathrm{mg}\right.$-eqv/dm $\left.{ }^{3}\right)$ & 1.41 & 0.28 & 0.95 & -0.26 & 0.26 & 0.99 \\
\hline $\mathrm{Mg}\left(8.5 \mathrm{mg}\right.$-eqv $\left./ \mathrm{dm}^{3}\right)$ & 0.78 & 2.80 & 0.99 & -0.21 & 0.09 & 0.96 \\
\hline
\end{tabular}

resin in the equilibrium state, $\mathrm{mg}$-eqv $/ \mathrm{cm}^{3} ; Q_{s}$ is the Langmuir constant (maximum capacity), $\mathrm{mg-eqv} / \mathrm{cm}^{3}$; and $K_{\text {ads }}$ is a constant that characterizes the "affinity" for the element being extracted.

This equation is linearized in the coordinates $1 / Q_{\mathrm{e}}-1 / C_{\mathrm{e}}$, and the linearized equation can then be used to find the values of $Q_{\mathrm{s}}$ (the intersection with the axis $1 / Q_{\mathrm{e}}$ ) and $K_{\text {ads }}$ (the slope tangent of the line).

In accordance with Freundlich's theory, the surface of an adsorbent is nonuniform, interaction takes place between adsorbed particles, and the active centers are not completely independent of one another. All this complicates the form of the equation of the isotherm. Freundlich showed that for a constant temperature the number of moles of an adsorbed dissolved substance per unit of mass of the adsorbent is proportional to the equilibrium concentration of the substance in the solution raised to a certain power that is always less than unity:

$$
Q_{\mathrm{e}}=K_{s} C_{\mathrm{e}}^{n}
$$

where $C_{\mathrm{e}}$ is the equilibrium concentration of an ion in the solution, mg-eqv/dm ${ }^{3}$, and $K_{S}$ and $n$ are the Freundlich constants.

The graph of the relation $\log Q_{\mathrm{e}}=f\left(\log C_{\mathrm{e}}\right)$ makes it possible to find the values of $K_{s}$ and $n$.

Figures 3 and 4 show the results of graphically constructing the sorption isotherm based on the Langmuir and Freundlich equations. It was established that the investigated models satisfactorily describe the experimental data, which is confirmed by the high values of the correlation coefficients $R^{2}$ (see Table 1). For low concentrations of the elements, the process of sorption on iminodiacetate ion-exchange resin Lewatit TP 207 is described best by the Langmuir equation.

The largest value $K_{\text {ads }}=211.25$ was obtained for zinc, which shows that the ion-exchange resin has the greatest affinity for this element. The selectivity series is as follows: $\mathrm{Zn}^{2+}>\mathrm{Ca}^{2+}>\mathrm{Mg}^{2+}$. This finding is consistent with the data obtained for iminodiacetate resins in general and Lewatit TP 207 in particular [7, 8].

However, the resin's capacity for zinc is only slightly greater than its capacity for calcium and magnesium. The constant $K_{s}$ in the Freundlich equation is also a quantitative measure of the affinity of a resin for the cation being extracted [9]. The values of this parameter are comparable for all of the cations (see Table 1), which indicates that the abilities of the resin to sorb zinc, calcium and magnesium are also quite comparable.

In addition, the limiting capacity of the resin increases from 0.26 to $1.41 \mathrm{mg}$-eqv/ $\mathrm{cm}^{3}$ for calcium and from 0.27 to $0.78 \mathrm{mg}$-eqv $/ \mathrm{cm}^{3}$ for magnesium with an increase in the initial concentration of the solution from $0.5 \mathrm{mg}$-eqv $/ \mathrm{dm}^{3}$ to 23.5 and $8.5 \mathrm{mg}$-eqv $/ \mathrm{dm}^{3}$, respectively. These increases in capacity are probably responsible for the competitive sorption of zinc and hardness cations that is seen under dynamic conditions.

\section{Conclusions}

1. The capacity of the ion-exchange resin increases with an increase in the equilibrium concentration of an element in the solution, which shows the affinity of the material for the investigated cations when they are being sorbed from monosolutions.

2. The process of extracting ionic zinc, calcium, and magnesium on ion-exchange resin Lewatit TP 207 is described quantitatively by the Langmuir and Freundlich sorption models. 
3. The selectivity of the resin for the investigated cations increases in the following order: $\mathrm{Zn}^{2+}>\mathrm{Ca}^{2+}>\mathrm{Mg}^{2+}$. There may also be significant sorption of hardness ions by the resin, particularly, when they are present in high concentrations (compared to zinc).

\section{REFERENCES}

1. V. A. Chanturiya, “Technology for recovering zinc from mine and quarry water,” Obogash. Rud., No. 1, 35-39 (2011).

2. A. B. Lebed, L. F. Akulich, and K. L. Timofeev, "Sorptive extraction of nonferrous metals from mine and quarry water," Proc. Int. Conf. New Technologies for the Concentration and Systematic Processing of Technogenic and LowDressability Mineral-Bearing Raw Materials (Plaksin Lectures 2011), Sept. 19-24, 2011, Verkhnyaya Pyshma, pp. 424-428.

3. $\quad$ R. S. Dave et al, "Removal of copper ions from electroplating waste water by weakly chelating anion exchange resins: Dowex 50 X 4, Dowex 50 X 2, and Dowex M-4195,” Der Pharma Chemica, No. 2 (2), $327-335$ (2010).

4. V. S. Soldatov and V. A. Bychkov, Ion-Exchange Resins in Multi-Component Systems [in Russian], Nauka i Tekhnika, Minsk (1988).

5. Yu. G. Frolov, Course in Colloid Chemistry. Surface Phenomena and Disperse Systems: Textbook for Higher Educational Institutions, 2nd edition, Khimiya, Moscow (1988).

6. A. A. Atia, A. M. Donia, and A. M. Yousif, "Comparative study on the recovery of silver(I) from aqueous solutions using different chelating resins derived from glycidyl methacrylate," J. Appl. Polym. Sci., 97, No. 3, 806-816 (2005).

7. R. Herring, Chelating Ion-Exchange Resins [Russian translation] (1971).

8. Information on the Product Lewatit MonoPlus TP-207, Lanxess Deutschland GmbH BU ION D-51369 Leverkusen, Vers. 2010-11-11, www.lenntech.com/products/resins/lanxess-lewatit/lanxess-lewatit-ion-resins.htm, accessed 10.25.2012.

9. L. A. Pimneva and E. L. Nesterova, "Sorption isotherm of ions of barium, copper, and yttrium on carboxyl cation exchange resin KB-4Pkh2," Sovr. Naukoem. Tekhnol., No. 4, 15-19 (2008). 